\title{
Tangence
}

\section{Comique et référence : Le Paon de Jules Renard et le Canard d'Alphonse Allais}

\section{Daniel Grojnowski}

Numéro 44, juin 1994

La référence littéraire

URI : https://id.erudit.org/iderudit/025815ar

DOI : https://doi.org/10.7202/025815ar

Aller au sommaire du numéro

Éditeur(s)

Tangence

ISSN

0226-9554 (imprimé)

1710-0305 (numérique)

Découvrir la revue

Citer cet article

Grojnowski, D. (1994). Comique et référence : Le Paon de Jules Renard et le Canard d'Alphonse Allais. Tangence, (44), 81-93.

https://doi.org/10.7202/025815ar d'utilisation que vous pouvez consulter en ligne.

https://apropos.erudit.org/fr/usagers/politique-dutilisation/ 


\section{Comique et référence : le Paon de Jules Renard et le Canard d'Alphonse Allais Daniel Grojnowski}

Le langage ne rend pas compte d'un "être là " des choses qui lui seraient extérieures, en ce sens il ne décrit pas le monde, matériel, imaginaire, conceptuel. Si l'actualisation du référent est possible ("Fermez la porte, s'il vous plaît"), si la confrontation entre le texte et le réel peut s'accomplir à l'occasion, il n'en reste pas moins qu'il revient au langage de construire le réel, une infinité de mondes divers qu'il nous donne à connaître et à lire. Au regard de l'analyse littéraire, le langage est toujours premier, constitutif de toutes sortes de réalités qui se réfèrent ou non aux expériences du lecteur.

Celui-ci, qu'il se trouve en présence d'une description de Zola ou d'une maxime de La Rochefoucauld, perçoit des procédures signifiantes, il est introduit dans un espace de communication où le message est moins soumis à l'épreuve de la vérité qu'à celle de la réception: une réception dont les aléas sont difficiles à raisonner. $\mathrm{Si}$, en effet, on peut évaluer la bonne intelligence d'un mode d'emploi à la réalisation de l'acte qu'il commande, comment estimer l'interprétation d'un texte littéraire qui, de toute évidence, ne cesse de donner matière à des lectures différentes?

Les causes d'une réception insatisfaisante sont innombrables: elle peut provenir d'une méconnaissance de la langue, des conventions, du genre, de l'époque, d'un style, etc. Comprendre les textes de Mallarmé, c'est avoir pris l'habitude de lire des poèmes, s'être imprégné de littérature symboliste, entretenir une familiarité avec les écrits de cet auteur, ses poésies, ses essais, sa correspondance. Le "comprendre", c'est adhérer à un mode d'expression, participer à l'acte de communication, en acceptant un langage qui se démarque des usages ordinaires, qui relègue à l'arrière-plan les contenus au profit d'une célébration du dire. Cette polarité "autotélique " de l'œuvre rend manifeste l'autonomie du langage sans que soit rompue toute attache avec la référence: même s'ils mettent en ouvre une déperdition maximale des contenus, les énoncés d'exception comme la glossolalie, les écrits *bruts", les 
82

poèmes dadaïstes ou expérimentaux, ceux de Mallarmé, ne cessent d'exploiter les savoirs du langage.

La plupart des écrits littéraires exigent du lecteur une imprégnation moindre que ces expressions extrêmes. Ils n'en supposent pas moins une compétence, un habitus. Il en va ainsi pour toute pratique culturelle, aussi rudimentaire qu'elle puisse paraître, comme la lecture des faits divers ou des chroniques sentimentales dans la presse populaire. C'est pourquoi l'analyse critique qui se consacre habituellernent à la compréhension des textes, tire également profit des interprétations défaillantes, insuffisantes ou aberrantes.

L'échec de la communication peut résulter d'une ignorance lexicale: "Tu m'achèteras des papayes" est incompréhensible pour qui ne connaît pas cette baie des Antilles (mais dans ce cas le dictionnaire peut réparer la défaillance). Il peut également résulter d'une référence à un savoir dont le lecteur ne dispose pas nécessairement ou à un savoir dont l'évidence s'estompe à mesure que s'écoulent les années. Tout écrit de circonstance, tout texte d'actualité, est ainsi menacé d'hermétisme involontaire du fait que sa référence (contexte, situation, événement de conjoncture) disparaît des mémoires, alors qu'il s'offre toujours à la lecture.

Le texte comique répond à une demande précise de lecteurs en mal d'amusement, qui le jugent sur pièce, sur son aptitude à faire rire ou sourire. Si bien qu'il se situe à l'écart des traditions réalistes, qu'il ne se soucie ni de vérité ni de vraisemblance. Lui importe pour l'essentiel de produire un effet. Il use de tous les stratagèmes, pourvu qu'il rende hilare. D'où le statut particulier de ses références: ou bien elles n'interviennent qu'au titre de fioriture ou bien elles doivent être comprises du plus grand nombre. Dans le premier cas, la référence joue un rôle accessoire, elle passe inaperçue aux moindres frais: simple indice de gaieté qui confirme une appartenance générique en laissant attendre le prochain rire à venir. Dans le second cas, elle joue un rôle important (voire déterminant comme dans les histoires drôles fondées sur un bon "mot") et elle prend valeur de clef: elle ouvre le texte aux significations, aux effets de sens auxquels il doit sa raison d'être. S'il lui advient de n'être pas comprise, l'édifice verbal qu'elle maintient, s'effondre tout entier. 


\section{Le Paon de Jules Renard}

L'histoire littéraire, l'érudition, s'efforcent de remédier aux déperditions du sens qu'entraîne l'oubli des références. Mais le texte comique, comme le mot d'esprit, ne peut s'appuyer sur un appareil de notes savantes sans risque de dénaturation. Quand ne jaillit pas l'étincelle de l'intelligence immédiate, l'explication fait long feu. On lira le portrait du Paon dans Histoires naturelles ${ }^{1}$ de Jules Renard de manière différente selon qu'on y repère une tradition littéraire ou qu'on y discerne également une référence d'époque.

\section{LE PAON}

Il va sûrement se marier aujourd'hui.

Ce devait être pour hier. En habit de gala, il était prêt. Il n'attendait que sa fiancée. Elle n'est pas venue. Elle ne peut tarder.

Glorieux, il se promène avec une allure de prince indien et porte sur lui les riches présents d'usage. L'amour avive l'éclat de ses couleurs et son aigrette tremble comme une lyre.

La fiancée n'arrive pas.

Il monte au haut du toit et regarde du côté du soleil. Il jette son cri diabolique:

\section{Léon! Léon!}

C'est ainsi qu'il appelle sa fiancée. Il ne voit rien venir et personne ne répond. Les volailles habituées ne lèvent même point la tête. Elles sont lasses de l'admirer. Il redescend dans la cour, si sûr d'être beau qu'il est incapable de rancune.

Son mariage sera pour demain.

Et, ne sachant que faire du reste de la journée, il se dirige vers le perron. Il gravit les marches comme des marches de temple, d'un pas officiel.

Il relève sa robe à queue toute lourde des yeux qui n'ont pu se détacher d'elle.

Il répète encore une fois la cérémonie.

Dans le premier cas, cette page met en scène le personnage du Glorieux. L'animal démarque l'humain à des fins satiriques. Le

1 La première édition des Histoires naturelles date de 1896. - Le paon * avait été publié dans L'écho de Paris du 25 octobre 1895. 
texte élabore son propre jeu de références par la double isotopie du "paon" et du "prince". Il hérite d'une tradition, celle du bestiaire, de la fable, de la satire, où les récits de La Fontaine interfèrent avec Les caractères de La Bruyère. Les références internes au texte qu'on lit, mettent également en place un implicite externe, d'obédience culturelle, qui relève de la longue durée, celui du portrait-charge.

Dans le second cas, la référence se précise. Elle vise la vogue de l'artifice et du raffinement "décadents". Le paon de Jules Renard n'apparaît pas comme un personnage chiffré mais comme l'emblème d'une préciosité en vogue, qui se passionne de travestissements exotico-romanesques, de japoniaiseries, de décors surchargés de nacre et de ramages, où se déploient plumes et palmes. Qu'on songe aux types pittoresques qu'incarnent le sâr Péladan, Jean Lorrain, Pierre Loti, qu'on se réfère à des Esseintes, aux illustrations de Beardsley ou aux portraits que brosse Boldini, peu importe. Pour un lecteur des premières éditions, "Le paon" se réfère manifestement à un esthétisme mâtiné d'inversion. Une photographie de Robert de Montesquiou (le futur poète des Paons), prise en 1887 dans son jardin de Passy, le montre se pavanant devant une ombrelle décorée, grande ouverte, qui lui fait faire la roue, littéralement ${ }^{2}$.

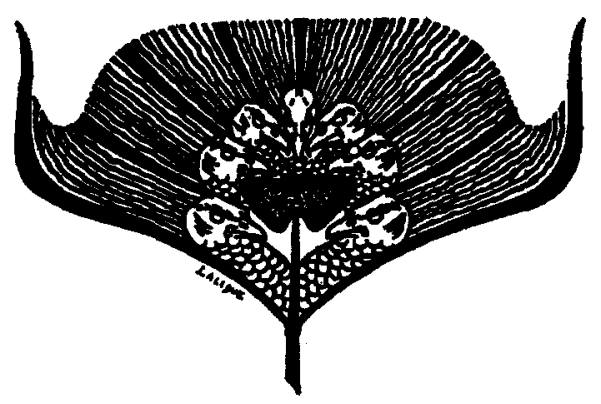

Dessin de Lalique pour la couverture du recueil Les paons (1901) de Robert de Montesquiou

2 Ce document est reproduit dans plusieurs ouvrages. Voir par exemple: Philippe Julian, Robert de Montesquiou, un Prince 1900, Librairie académique Perrin, 1965, dossier photographique, p. 3. 
La référence culturelle indistincte se double ainsi d'une référence datée. Le type de l'esthète homosexuel apporte au portrait du Paon "glorieux" (c'est-à-dire "orgueilleux", "vaniteux", dans la langue du XVII siècle) une valeur ajoutée. Elle transforme la tonalité du texte aussi bien que son contenu: le portrait-charge vire à l'acide, tandis que toutes sortes d'informations se trouvent motivées par l'émergence d'une composante nouvelle: on comprend désormais de quel genre de "fiancée" il s'agit, pourquoi le Paon l'appelle "Léon!", pourquoi les volailles poulettes se désintéressent de l'affaire et pourquoi chaque fois le mariage est remis au lendemain.

L'humour en demi-teinte de Jules Renard appelle toutes sortes d'accommodations de la part des lecteurs. La gamme des effets produits est fonction de ses compétences en matière littéraire, historique, mais aussi en matière d'usage de cour ou de bassecour. Il n'en demeure pas moins que s'y affirme clairement l'appartenance du texte à la Littérature. Le lecteur qui ne perçoit pas l'allusion au dandysme "fin de siècle ", outre qu'il s'amuse à imaginer un Fat célibataire, goûte le poème en prose et ses effets de plume.

\section{Le Canard d'Alphonse Allais}

Le petit conte qu'Alphonse Allais publie dans Le cbat noir en février 1890 et qu'il reproduit dans son premier recueil, $\bar{A}$ se tordre, pose un problème plus délicat en matière de référence ${ }^{3}$. Il se démarque des Belles-Lettres par un parti pris de désinvolture: le récit renonce au bel appareillage auquel nous accoutume la tradition littéraire. Rédigé par un "auteur gai ", il doit soutenir sa réputation. Toutefois il use d'une dénomination, il pose une problématique qui ne manquent pas de dérouter les lecteurs du temps et les lecteurs d'aujourd'hui, pour des raisons différentes:

3 Le chat noir, $1^{\mathrm{er}}$ février 1990 . À se tordre, *histoires chatnoiresques ", Ollendorff, 1891. Dans la revue, le conte est dédié à Georges Courteline. La dédicace disparaît dans le recueil. Au troisième paragraphe nous reproduisons la version du Chat noir dont la leçon apparaît plus satisfaisante que celle des Euvres anthumes de François Caradec (coll. "Bouquins", Robert Laffont) : "le marquis de Belveau, président du comité d'administration de la Société..." (et non: "du comité d'organisation de la Société.... *). 


\section{FERDINAND}

Les bêtes ont-elles une âme? Pourquoi n'en auraient-elles pas? J'ai rencontré, dans la vie, une quantité considérable d'hommes, dont quelques femmes, bêtes comme des oies, et plusieurs animaux pas beaucoup plus idiots que bien des électeurs.

Et même - je ne dis que le cas soit très fréquent - jai personnellement connu un canard qui avait du génie.

Ce canard, nommé Ferdinand, en l'honneur du grand Français, était né dans la cour de mon parrain, le marquis de Belveau, président du comité d'administration de la Société générale d'affichage dans les tunnels.

C'est dans la propriété de mon parrain que je passais toutes mes vacances, mes parents exerçant une industrie insalubre dans un milieu confiné.

(Mes parents - j'aime mieux le dire tout de suite, pour qu'on ne les accuse pas d'indifférence à mon égard - avaient établi une raffinerie de phosphore dans un appartement du cinquième étage, rue des Blancs-Manteaux, composé d'une chambre, d'une cuisine et d'un petit cabinet de débarras, servant de salon.)

Un véritable éden, la propriété de mon parrain! Mais c'est surtout la basse-cour où je me plaisais le mieux, probablement parce que c'était l'endroit le plus sale du domaine.

Il y avait là, vivant dans une touchante fraternité, un cochon adulte, des lapins de tout âge, des volailles polychromes et des canards à se mettre à genoux devant, tant leur ramage valait leur plumage.

Là, je connus Ferdinand, qui, à cette époque, était un jeune canard dans les deux ou trois mois. Ferdinand et moi, nous nous plûmes rapidement.

Dès que j'arrivais, c'étaient des coincoins de bon accueil, des frémissements d'ailes, toute une bruyante manifestation d'amitié qui m'allait droit au cœur.

Aussi l'idée de la fin prochaine de Ferdinand me glaçait-elle le coeur de désespoir.

Ferdinand était fixé sur sa destinée, conscius sui fati. Quand on lui apportait dans sa nourriture des épluchures de navets ou des cosses de petits pois, un rictus amer crispait les commissures de son bec, et comme un nuage de mort voilait d'avance ses petits yeux jaunes. 
Heureusement que Ferdinand n'était pas un canard à se laisser mettre à la broche comme un simple dindon: "Puisque je ne suis pas le plus fort, se disait-il, je serai le plus malin", et il mit tout en ceuvre pour ne connaître jamais les hautes températures de la rotissoire ou de la casserole.

Il avait remarqué le manège qu'exécutait la cuisinière, chaque fois qu'elle avait besoin d'un sujet de la basse-cour. La cruelle fille saisissait l'animal, le soupesait, le palpait soigneusement, pelotage suprême!

Ferdinand se jura de ne point engraisser et il se tint parole.

Il mangea fort peu, jamais de féculents, évita de boire pendant ses repas, ainsi que le recommandent les meilleurs médecins. Beaucoup d'exercice.

Ce traitement ne suffisant pas, Ferdinand, aidé par son instinct et de rares aptitudes aux sciences naturelles, pénétrait de nuit dans le jardin et absorbait les plantes les plus purgatives, les racines les plus drastiques.

Pendant quelque temps, ses efforts furent couronnés de succès, mais son pauvre corps de canard s'habitua à ces drogues, et mon infortuné Ferdinand regagna vite le poids perdu.

Il essaya des plantes vénéneuses à petites doses, et suça quelques feuilles d'un datura stramonium qui jouait dans les massifs de mon parrain un rôle épineux et décoratif.

Ferdinand fut malade comme un fort cheval et faillit y passer.

L'électricité s'offrit à son âme ingénieuse, et je le surpris souvent, les yeux levés vers les fils télégraphiques qui rayaient l'azur, juste au-dessus de la basse-cour, mais ses pauvres ailes atrophiées refusèrent de le monter si haut.

Un jour, la cuisinière, impatientée de cette étisie incoercible, empoigna Ferdinand, lui lia les pattes en murmurant : «Bah! à la casserole, avec une bonne platée de petits pois!...*

La place me manque pour peindre ma consternation.

Ferdinand n'avait plus qu'une seule aurore à voir luire.

Dans la nuit je me levai pour porter à mon ami le suprême adieu, et voici le spectacle qui s'offrit à mes yeux :

Ferdinand, les pattes encore liées, s'était traîné jusqu'au seuil de la cuisine. D'un mouvement énergique de friction alternative, il aiguisait son bec sur la marche de granit. Puis, d'un coup sec, il coupa la ficelle qui l'entravait et se retrouva debout sur ses pattes un peu engourdies. 
88

Tout à fait rassuré, je regagnai doucement ma chambre et m'endormis profondément.

Au matin, vous ne pouvez pas vous faire une idée des cris remplissant la maison. La cuisinière, dans un langage malveillant, trivial et tumultueux, annonçait à tous la fuite de Ferdinand:

- Madame! Madame! Ferdinand qui a fichu le camp!

Cinq minutes après, une nouvelle découverte la jeta hors d'ellemême :

- Madame! Madame! Imaginez-vous qu'avant de partir, ce cochon-là a boulotté tous les petits pois qu'on devait lui mettre avec!

Je reconnaissais bien, à ce trait, mon vieux Ferdinand.

Qu'a-t-il pu devenir, par la suite?

Peut-être a-t-il appliqué au mal les merveilleuses facultés dont la nature, alma parens, s'était plu à le gratifier.

Qu'importe? Le souvenir de Ferdinand me restera toujours comme celui d'un rude lapin.

Et à vous aussi, j'espère!

"Ferdinand" manifeste une liberté d'allure qui le situe aux antipodes de la belle tenue dont "Le paon" de Jules Renard ne se départit pas. Le narrateur d'Alphonse Allais ne refuse ni la plaisanterie facile, ni le clin d'oil égrillard, ni la digression, ni le pur verbiage, ni la cuistrerie. Il joue sur tous les tableaux, fait flèche de tout bois. Son patchwork démarque le conte philosophique, la fable enfantine, le récit d'aventure, le monologue de cabaret. Un personnage entre en scène et raconte son histoire, tambour battant. Épisodes et développernents se succèdent sans grand souci de cohérence. Ils scandent une action qui semble se construire au fur et à mesure qu'elle s'énonce. La sagesse populaire côtoie la confidence, les conseils médicaux, l'épisode à suspens. D'un registre à l'autre s'improvise un petit conte qui se consacre pour l'essentiel au plaisir de dire. Seul le lecteur réfractaire relèvera que Ferdinand, le héros, souffre d'étisie (ce terme désigne toute maladie qui provoque un amaigrissement excessif) bien qu'il ait repris du poids - ou se demandera pourquoi, une fois délivré de ses liens, il devrait s'adonner "au mal" (si c'est par esprit de vengeance, le conteur ne prend nul soin de l'indiquer).

Mais la désinvolture atteint son comble avec la phrase initiale qui oriente le récit: "Les bêtes ont-elles une âme?" Par cette 
question, l'auteur semble reprendre à son compte un problème philosophique de large audience, auquel La Fontaine a consacré les dernières pages du neuvième livre de ses Fables. Dans le "Discours à Madame de la Sablière ", il conteste la théorie des animaux-machines, chère à Descartes, par les exemples du cerf aux abois, de la perdrix qui protège ses petits, de la société des castors ou des ruses guerrières qu'imaginent les renards. Ses conclusions font suite à la fable "Les deux rats, le renard et l'œuf ": non seulement les animaux ne sont pas mus par d'“aveugles ressorts" mais ils s'apparentent à l'Homme. Si bien que La Fontaine émet l'hypothèse d'une première âme commune aux

Sages, fous, enfants, idiots,

Hôtes de l'univers sous le noms d'animaux ${ }^{4}$

et d'une deuxième âme où perce la "raison" qui seule distingue l'Homme, et qui manifeste sa divinité. Ainsi quelque "quintessence d'atome " ou "extrait de lumière" autorise, selon lui, à évoquer une âme propre aux animaux.

Alphonse Allais aborde le problème de manière directe pour l'abandonner aussitôt et l'oublier sans retour. Si "Ferdinand" démontre, sur le mode badin, comment se manifeste l'intelligence d'un canard de ferme, il ne donne pas réponse à la question qui est censée informer le récit. Arrivé à la fin, le lecteur ne peut dire si l'"âme" participe aux facultés merveilleuses dont la nature a doué Ferdinand. L'enjeu théologique s'est dissipé au profit d'un jeu essentiellement verbal.

Délaissant la composante théologique du problème traité par La Fontaine, Alphonse Allais compare l'Animal à l'Homme dans une fable de sa façon. Il substitue à l'argumentation logique une logique argumentative d'un autre ordre, en refusant de se placer sur le terrain des "idées". Il utilise le seul instrument qui lui soit familier, celui des mots. Son récit révèle les relations d'équivalence qu'établit le langage - dans des formules stéréotypées ou des expressions de circonstance - entre les deux espèces. Exposé dès le premier paragraphe, ce thème ne cesse de nourrir la trame narrative: si les hommes (et les femmes) peuvent se révéler "bêtes comme des oies", les animaux, quant à eux, ne sont pas plus "bêtes" que des électeurs.

4 La Fontaine, "Les deux rats, le renard, et l'œuf ", v. 44-45. 
90

Le héros de l'histoire, Ferdinand le canard, joue un rôle décisif dans cette démonstration, du fait qu'il porte un nom d'homme sans jamais trahir son identité palmipède. Situé au centre d'une double série lexicale qui assimile l'Homme à l'Animal, il relève lui-même des deux espèces. Sa double appartenance est avérée par la multiplicité des expressions auxquelles il se trouve associé. De toute évidence, la prolifération fait preuve:

\section{L'Homme et l'Animal vs l'Animal et l'Homme}

- hommes et femmes: "bêtes comme des oies"

- marquis: Belveau

- la cuisinière : [rosse]

- animaux: "pas beaucoup plus bêtes que des électeurs"

\section{Ferdinand}

A. Animal: canard

dindon ("comme un simple dindon")

cheval ("comme un fort cheval")

cochon ("ce cochon-là ")

lapin ("un rude lapin")

B. Homme: le "grand Français" [Ferdinand de Lesseps]

Le canard héros porte le nom d'un être humain. Il génère le cortège des comparaisons animales qui le caractérisent (Ferdinand: "un rude lapin"). Il suscite par ailleurs une dénomination conforme au principe de double appartenance (le marquis de Belveau), tout en suggérant d'autres comparaisons de même régulation (par exemple: la cuisinière est une belle rosse). La divagation lexicale s'égrène de manière anarchique, tout en réitérant le principe d'équivalence qui pose la thèse du petit conte moral.

Reste à raisonner une référence qui risque aujourd'hui de passer inaperçue auprès de nombreux lecteurs. Manifeste pour ceux des années 1890 , elle a dû cependant mettre en difficulté certains d'entre eux: pourquoi le héros de l'histoire porte-t-il le prénom de celui que Gambetta avait appelé un "grand Français", Ferdinand de Lesseps ${ }^{5}$ ?

5 C'est Gambetta qui a prononcé cette formule à la fin d'un banquet, en 1879. Depuis elle accompagne souvent le nom de Ferdinand de Lesseps. 
Au moment où paraît le conte d'Alphonse Allais, le célèbre diplomate est âgé de quatre-vingt-six ans. Il est celui qui a surmonté, vingt ans plus tôt, d'innombrables obstacles économiques, techniques ou diplomatiques pour réaliser le canal de Suez: son inauguration, en 1869, lui a successivement ouvert les portes de l'Académie des sciences puis de l'Académie française. En 1880, il récidive en fondant la Compagnie universelle du canal interocéanique de Panama, entreprise quí lui vaut une série de déboires et qui finit par être mise en liquidation (février 1889). S'il faut attendre 1892 pour qu'une campagne de presse menée par la droite révèle la collusion entre les milieux d'affaires et les milieux politiques, depuis 1889 nul n'ignore les menaces qui pèsent sur les actionnaires de la Compagnie ${ }^{6}$. Chaque semaine dans Le chat noir, un "Bulletin financier" (signé Marsoin) dénonce ses pratiques illégales ainsi que les informations mensongères de Ferdinand de Lesseps qu'il taxe de sénilité (16 février 1889). Si bien qu'au regard du lecteur de l'époque, le personnage d'Alphonse Allais se charge de suggestions diverses:

- La campagne de presse qu'a menée Ferdinand de Lesseps pour attirer les capitaux se révèle un beau "canard".

- Ferdinand de Lesseps est une vieille "bête", son entreprise est boîteuse, elle bat de l'aile, elle a fait un beau "couac".

- Il va prendre le "bouillon", il va passer à la "casserole" (de fait Ferdinand de Lesseps et son fils seront condamnés en février 1893 à cinq ans de prison et trois mille francs d'amende, condamnation qui sera annulée par la Cour de cassation).

Ou encore :

- Ferdinand de Lesseps est un malin qui saura se tirer d'affaire. Il trompera la vigilance de ceux qui pensent n'en faire qu'une bouchée.

Alphonse Allais désamorce la référence - exprimée en clair - à Ferdinand de Lesseps. Il neutralise les implications du récit à l'actualité pour les transposer en une aventure qui n'a, en apparence, ni queue ni tête. Au lieu de s'exercer à la satire que peuvent attendre des lecteurs du temps, il brode une historiette farfelue. Toutefois la question posée en incipit trouve en fin de

6 Voir Jean Bouvier, Les deux scandales de Panama, Gallimard, coll. "Archives n, 1964. 
compte sa réponse: le jeu verbal permet au narrateur de retomber sur ses pattes. La référence à Ferdinand de Lesseps et à son fameux canal permet d'énoncer la morale de l'histoire par la grâce du calembour. Si, en effet, Ferdinand de Lesseps est responsable de l'entreprise et de la faillite du canal de Panama, cela ne prouve en rien que Ferdinand le canard pas d'âme n'a...

Dans les exemples du "Paon" et de "Ferdinand", les références à l'actualité informent le texte comique. Elles lui confèrent une part appréciable de sa saveur. Elles jouent un rôle important tout en demeurant soit implicites (Jules Renard) soit fugitives, énigmatiques (Alphonse Allais). Mais dans tous les cas, le contexte daté informe un texte dont Gérard Genette dirait: "Son être tient à son site ${ }^{7}$. Faute d'en percevoir les références, le lecteur ne le "comprend" pas, il n'en percoit pas la saveur: l'effet comique fait long feu.

Cependant la signification ne découle pas d'un simple décryptage. Dans la trame du texte s'entrecroisent des références à l'actualité, des références internes (le Paon/les volailles; le canard / la cuisinière) mais aussi des références propres à la réception. Le lecteur élabore le sens en fonction d'un savoir dont la pertinence n'est pas toujours assujettie à l'autorité du texte: lire, c'est aussi construire un sens plausible, interpréter l'écrit en fonction de savoirs diffus et selon des parcours dont nul ne contrôle le tracé. En ce sens la référence se situe au croisement d'effets qui en déterrninent les valences multiples.

Même dans le cas particulier où une interprétation est avérée (par ébauche, brouillon, variante, lettre ou conversation, par exemple), l'interprétation ne peut être imposée, la prolifération sémiotique n'est pas interrompue. Le lecteur dispose librement de ses droits qui incluent celui du contre-sens ou de la valeur ajoutée. Rien ne prouve, après tout, que le paon représente l'esthète homosexuel (dans son Joumal, Jules Renard compare Robert de Montesquiou à un oiseau de proie qui se nourrit de vanité ${ }^{8}$ ) ni que le calembour souterrain de "Ferdinand" ait été concerté par

7 Gérard Genette, Poétique, n 69, février 1967, p. 3.

8 "Schwob me présente à Montesquiou, qui a une figure vieillie et dit: "Très flatté", du bout d'un bec d'oiseau de proie qui ne se nourrirait que de vanités." Jules Renard, Journal, 10 décembre 1896, Gallimard, "Bibliothèque de la Pléiade, 1960 , p. 363. 
l'auteur (on ne prête qu'aux riches) ${ }^{9}$. Si les références construites par l'interprétation confèrent au texte comique sa pleine saveur, on peut se demander ce qu'il devient, une fois privé de ces grains de sel, de ses grains de folie.

Ainsi comprise, la référence comporte des plans distincts, celui du texte, du contexte, de sa réception. Mais dans tous les cas - et tout particulièrement quand il s'agit du "comique " qui provoque une réaction perceptible : rire ou sourire - le texte est à prendre comme un tout, il communique d'un seul tenant. D'où son extrême fragilité, le caractère aléatoire de ses effets. Denrée périssable, sa valeur est toujours fonction d'un public, d'une aire et d'une date de consommation.

9 J'ai interrogé les deux meilleurs spécialistes d'Alphonse Allais au sujet de la dénomination du canard. Selon François Caradec elle s'explique par la présence de Ferdinand de Lesseps au thêâtre d'ombres du Chat Noir. Quant à Jean-Marc Defays, il m'écrit : "J'ai lu et relu le "Ferdinand" qui vous intrigue, et, non, vraiment, je ne comprends pas l'allusion qu'il contient à propos de de Lesseps. " 